\title{
Forward and small-x physics at CMS
}

\author{
Paolo Gunnellini ${ }^{1 a}$ \\ on behalf of the CMS Collaboration \\ ${ }^{1}$ Deutsches Elektronen-Synchrotron (DESY) Notkestrasse 85, 22607, Hamburg (Germany)
}

\begin{abstract}
A review of experimental measurements from the Compact Muon Solenoid Experiment at the LHC Run 1 is presented. A particular focus is given to jet results in the central and forward regions, and to measurements sensitive to the behaviour of small- $x$ gluons inside the proton and to double parton scattering.
\end{abstract}

\section{Introduction}

The study of the dynamics of hadron jets in proton-proton collisions yields important information about underlying partonic processes. The production of jets with large transverse momenta $\mathrm{p}_{T}$ is thought to be originated from scatterings of partons which can be described by the theory of strong interactions. The cross section of such a proton-proton collision process is factorized in a convolution of the cross section for a partonic subprocess and the parton distribution functions (PDF). The QCD semi-hard interactions experienced by quarks and gluons are treated analytically through the so-called parton shower calculations. Different approaches to perform the parton evolution have been developed, depending on which kinematic region of the phase space is probed and several evolution equations are available for the calculations. At large splitting scales $Q^{2}$ and not too large longitudinal momentum fraction $x$, the Dokshitzer-Gribov-Lipatov-Altarelli-Parisi (DGLAP) equations [5] are good tools to calculate the partonic evolution with great success in reproducing a wide range of measurements. In the regime of low- $x$, though, the approximations used by the DGLAP approach break down and the formalism needs to be improved by the Balitsky-Fadin-Kuraev-Lipatov (BFKL) evolution scheme. Furthermore, at high factorization scales, the gluon distributions become large at low values of longitudinal momentum fractions of the colliding protons. In this regime, on the one hand parton recombination, i.e. saturation (see e.g. [1]), may occur. On the other hand, the probability to have more than one partonic interaction becomes non-negligible, leading to the production of pairs of physics objects at high transverse momentum via double parton scattering (DPS).

\section{Jet measurements}

At the Large Hadron Collider (LHC), various jet measurements, such the inclusive [2, 3] or the multijet [4] cross section for high $\mathrm{p}_{T}$ jets, have been measured at a collision energy $\sqrt{s}=7 \mathrm{TeV}$ by the CMS experiment and are in good agreement with predictions obtained at next-to-leading order (NLO)

ae-mail: paolo.gunnellini@desy.de 
quantum chromodynamics (QCD). This good description of data represents a nice success of the current formalism used for calculating partonic interactions in pp collisions. Many attempts have been tried in multijet measurements to reach regions of the phase space where BFKL effects might appear but so far, no stringent evidence has been observed yet. In particular, by looking at more sophisticated scenarios than inclusive jets, e.g. by measuring jets at high pseudorapidities or by requiring correlations among selected jets, one has the possibility to be sensitive to lower and lower $x$ values. Some of these scenarios have been measured by the CMS experiment to investigate different evolution models and are presented in the following.

The single-jet cross section has been measured [6] for the two leading, i.e. with highest $p_{T}$, jets in inclusive dijet events containing at least one forward (in the pseudorapidity range $3.2<|\eta|<4.7$ ) and one central jet (defined by the region $|\eta|<2.8$ ). The data are based on $3.14 \mathrm{pb}^{-1}$ of integrated luminosity at $\sqrt{s}=7 \mathrm{TeV}$. Jets are reconstructed using the anti- $k_{T}$ algorithm $(\mathrm{R}=0.5)$ in the $p_{T}$ range $35-150 \mathrm{GeV}$. The single-jet cross section is shown in Figure 1, as a function of the $p_{T}$ of the forward jet. The total systematic uncertainties are of the order of $20-30 \%$, dominated by the absolute jet energy scale. The measurement, compared to perturbative QCD calculations, as implemented in the parton-shower event generators PYTHIA [7, 8] and HERWIG++ [9] at leading order (LO) or POWHEG+PYTHIA [10] at NLO, is found to be in agreement within the current experimental and theoretical uncertainties. Predictions from Monte Carlo (MC) event generators implementing different kinds of parton evolution, such the combined DGLAP+BFKL resummation model of CASCADE [11], are also found to be in good agreement with the measured dijet cross section.
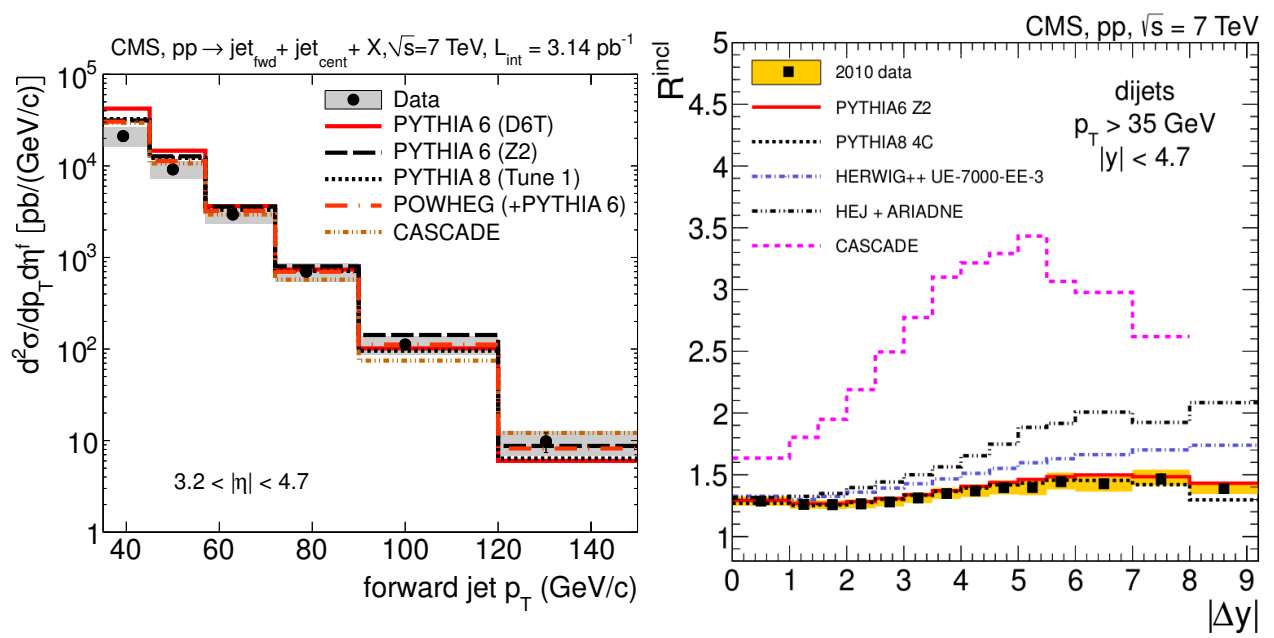

Figure 1. Differential cross sections as a function of the forward jet $p_{T}$ for dijet events with at least one central jet and one forward jet, compared to predictions from several models (left). Ratios of the inclusive to exclusive dijet cross sections as a function of the rapidity separation $\Delta y$ between the two jets compared to the predictions of the DGLAP-based MC generators PYTHIA6, PYTHIA8 and HERWIG++, as well as of CASCADE and HEJ+ARIADNE which incorporate elements of the BFKL approach (right).

A production of at least two jets is also studied in pp collisions at $\sqrt{s}=7 \mathrm{TeV}$ as function of the rapidity separation $\Delta y$ between the jets [12], for jets with $\mathrm{p}_{T}>35 \mathrm{GeV}$ and $|y|<4$.7. Two classes of events are considered: 
- inclusive events, with at least one pair of jets;

- exclusive events, with exactly one pair of jets.

The ratio $\mathrm{R}^{\mathrm{incl}}$ of the cross section of all pairwise combinations of jets from the inclusive sample to the exclusive dijet cross section is shown on Figure 1(right) as a function of $\Delta y$. This observable is expected to be sensitive to effects beyond collinear factorization, as the radiation probability increases with $|\Delta| \mathrm{y}$. The predictions of the MC event generators PYTHIA6 and PYTHIA 8 agree with the measurements, while predictions from the HERWIG++ event generator exhibit a more pronounced rise with $|\Delta y|$. The BFKL-motivated generators CASCADE and HEJ+ARIADNE [13] predict for these ratios a significantly stronger rise than observed.

Finally, for a further investigation of possible BFKL effects, correlations in azimuthal angle of the most forward and most backward in rapidity jets, (the so-called Mueller-Navelet jets) are measured with data collected in pp collisions with the CMS detector at the LHC at $\sqrt{s}=7 \mathrm{TeV}$. The azimutalangle distributions between Mueller-Navelet jets are measured for jets with $p_{T}>35 \mathrm{GeV}$ and $|y|<4.7$. The results are presented as a function of the difference in azimuthal angle between the two selected jets in different ranges of rapidity separation $\Delta y$, reaching a $\Delta y$ up to 9.4 in Figure 3. The results are compared to predictions of various Monte-Carlo event generators and analytical predictions based on the DGLAP and BFKL parton evolution schemes.
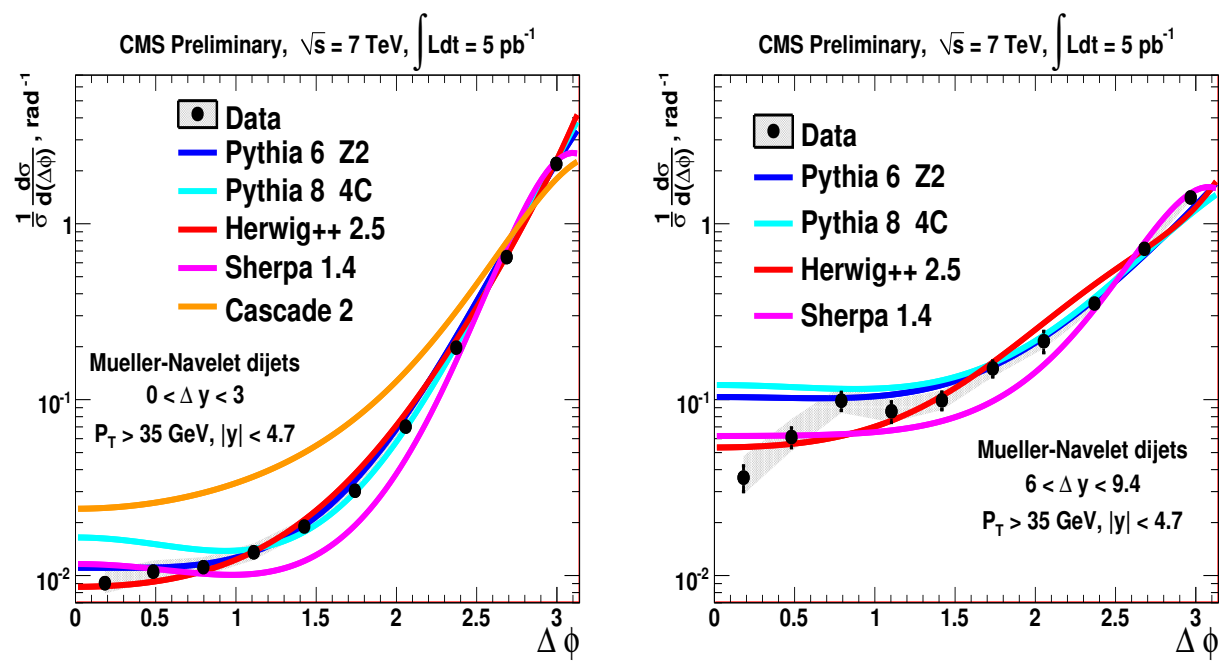

Figure 2. Distribution of azimuthal angle difference, $\Delta \phi$, between $\mathrm{MN}$ jets in rapidity interval $\Delta y<3.0$ (left) and $6<\Delta y<9.4$ and its comparison to the predictions from LL based MC generators PYTHIA 6 tune Z2, PYTHIA 8 tune 4C, HERWIG++ 2.5, SHERPA 1.4 and LL BFKL motivated MC generator CASCADE.

From these measurements, no clear evidence of BFKL effects or of measurements deviating from DGLAP evolution scheme is obtained. MC event generators which are based on the DGLAP formalism are able to reproduce well a wide range of jet measurements. On the one hand, this might be due to the fact that the sensitivity to sufficiently small $x$ values is not yet achieved reached at $\sqrt{s}$ $=7 \mathrm{TeV}$; if this is the case, measurements at a higher collision energy during LHC RunII will scan a still unexplored phase space which might reveal BFKL signals. On the other hand, BFKL effects might be hidden in the measured phase space due to many background contributions. It is indeed very 
difficult to select and isolate a "pure" gluonic ladder in a regime where the BFKL scheme should rule the parton evolution. At high $p_{T}$, the DGLAP approach is expected to dominate, while for decreasing $p_{T}$, increasing activity from MPI contributes to the final state. Independently on the $p_{T}$ threshold, quark ladders following a DGLAP-based evolution add a major background and make the search of BFKL effects very complicated. New strategies for tackling these issues are under discussion and are summarized in the following:

- measurements of more exclusive scenarios, e.g. rapidity gap in dijet inclusive or exclusive ratios [14];

- measurements of more inclusive scenarios selecting corners of the phase space, e.g. jets in the forward region, in final states with a high jet multiplicity or associated to heavy-flavour production;

By pursuing these ideas, the new LHC phase will surely be an exciting and vivid period for searching BFKL effects.

\section{Small-x gluon studies}

A large set of measurements has been also performed by the CMS experiment for investigation of the dynamics of gluons at small longitudinal fractional momenta $x$. The integrated yields of events with a leading charged particle or a leading charged-particle jet with $p_{T}$ above a given threshold have been measured in pp collisions at $\sqrt{s}=8 \mathrm{TeV}$ in a data sample corresponding to an integrated luminosity of $45 \mu \mathrm{b}^{-1}$ [15]. The yields are found to be relatively flat in the $p_{T}$ region around $1 \mathrm{GeV}$ (where the fixed-order perturbative parton-parton cross section diverges in the absence of any mechanism that saturates or unitarises the perturbative QCD scattering), followed by a steep decrease for $p_{T}>$ $10 \mathrm{GeV}$. The flattening behaviour observed at very low $p_{T}$ is reproduced by models which apply a cutoff of the partonic cross section at values between 3 and $5 \mathrm{GeV}$, with the best description provided by the EPOS event generator [16]. Further comparisons of this measurement with various other phenomenological predictions of hadronic interaction models will help to improve the description of the transition between the perturbative and non-perturbative QCD regimes, which is believed to be dominated by the effects of parton density saturation and MPI.

A study at generator level, aiming for an investigation of the small- $x$ gluon behaviour, has shown an interesting feature. Even though demonstrated only at a qualitative level, an increase of the gluon density at small- $x$ values tends to be preferred for a consistent description of measurements at central and forward pseudorapidities. In particular, it has been observed that the description of charged-particle multiplicity at high pseudorapidities [17] provided by tunes using the NNPDF2.3LO PDF [18], e.g. Monash [19] and CUETP8M1 [17], is better than tunes using other PDF sets. This is due to the different small- $x$ behaviour between NNPDF2.3LO and the other PDF. In fact, at low parton scales $\mathrm{Q}^{2}$, the NNPDF2.3LO PDF has a gluon distribution at small $x$ values that is larger than in HERAPDF1.5LO [20]. This contributes to more particles (and more energy) in the forward region. An investigation performed within the CMS collaboration confirmed this conclusion. By taking the HERAPDF1.5LO PDF set as a default, the low-x gluon distribution has been artificially increased according to a function (see Figure 1(left)) with two free parameters, $x_{0}$ and $p_{1}$.

The $x_{0}$ parameter sets the initial point of the $x$ gluon increase, while the steepness of the rise is ruled by the $p_{1}$ parameter. The nominal gluon distribution in HERAPDF1.5LO and various distributions obtained by applying different modifications are shown in Figure 4(left). A tune based on a parton distribution function using $x_{0}=2 \cdot 10^{-5}$ and $p_{1}=5$, resulted to improve the description of charged-particle multiplicity consistently at both central and forward pseudorapidities (see Figure 4(right)) with respect to the tune CUETP8S1 [17] based on the nominal HERAPDF1.5LO PDF 

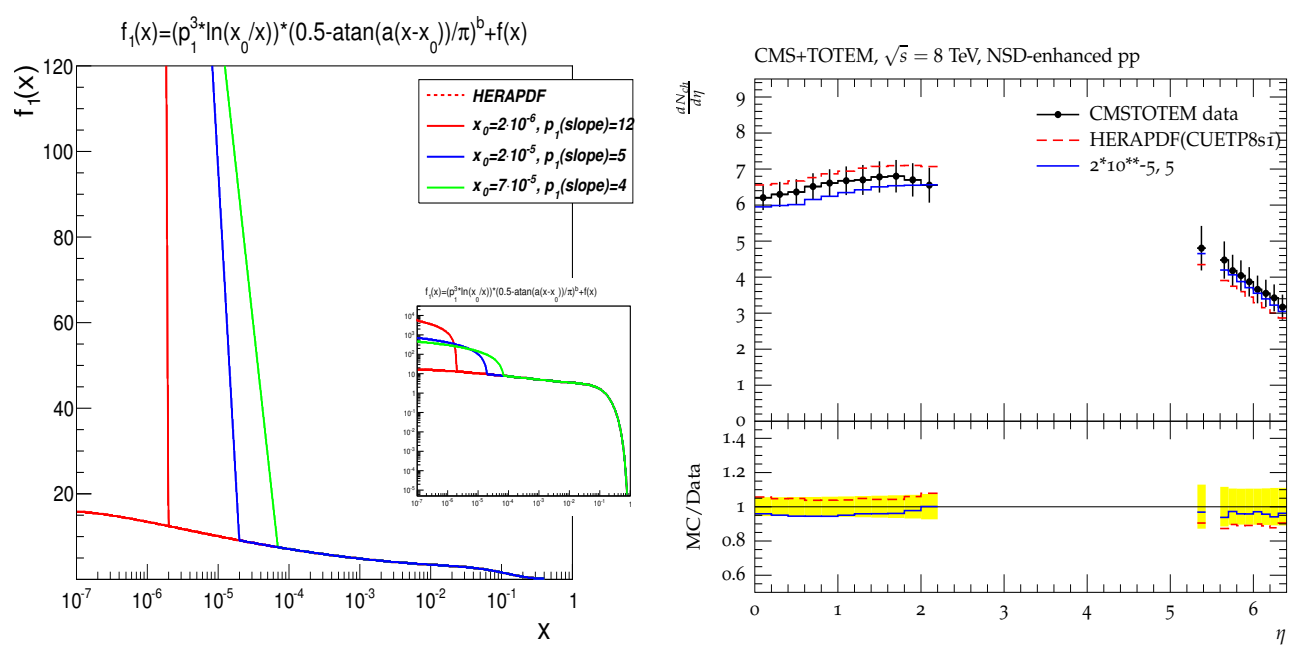

Figure 3. Gluon distribution as a function of the longitudinal fractional momentum $x$ for the nominal HERAPDF1.5LO PDF set and for the modified sets with different values of $x_{0}$ and (left). Comparison of charged-particle multiplicity as a function of $\eta$ for the CUETP8S1-HERAPDF tune and a tune performed with modified low- $x$ gluon behaviour (right).

set. Note that the range of modified $x$ values in the considered gluon distributions are below the range of the HERA deep inelastic scattering data. Future measurements sensitive to this low- $x$ regime are strictly needed to confirm the results of this qualitative study and to further constrain the behaviour of the gluon density.

\section{Double parton scattering measurements}

A wide range of measurements sensitive to DPS contributions has been also carried out within the CMS physics program for different physics channels. The investigated channels are characterized by a high number of physics objects in the final state, e.g. jets. Measurements related to the W+twojet [21], four-jet [22], $\gamma+$ three-jet [23] and two-b+two-light-jet [24] final states have been released by the CMS experiment. The current strategy for DPS-extraction analyses is based on the association of the selected objects in two pairs and on the measurement of differential cross sections as a function of correlation observables defined by the kinematical topology of the final state. The two pairs can be produced by two independent scatterings in case of a DPS event and the correlation observables are expected to be able to disentangle this occurrence. Studies at generator level have shown that variables which are most sensitive to a DPS contribution are the ones which consider the relative topology of all selected objects, not only of the objects of one of the two pairs [25]. One of such observables is $\Delta \mathrm{S}$, which corresponds to the azimuthal angle between the two selected pairs and is defined as:

$$
\Delta \mathrm{S}=\arccos \left(\frac{\vec{p}_{\mathrm{T}}\left(\text { pair }_{1}\right) \cdot \vec{p}_{\mathrm{T}}\left(\text { pair }_{2}\right)}{\mid \vec{p}_{\mathrm{T}}\left(\text { pair }_{1}\right)|\cdot| \vec{p}_{\mathrm{T}}\left(\text { pair }_{2}\right) \mid}\right)
$$

For the $\mathrm{W}+$ two-jet channel, pair ${ }_{1}$ and pair $_{2}$ are, respectively, the reconstructed $\mathrm{W}$ and the dijet system, while in the four-jet (two-b+two-light-jet) final state, they correspond to the hard (bottom) 
and soft (light) dijet systems. In Figure 4, the differential cross section normalized to the number of selected events as a function of the $\Delta \mathrm{S}$ observable measured by CMS is shown for various final states.
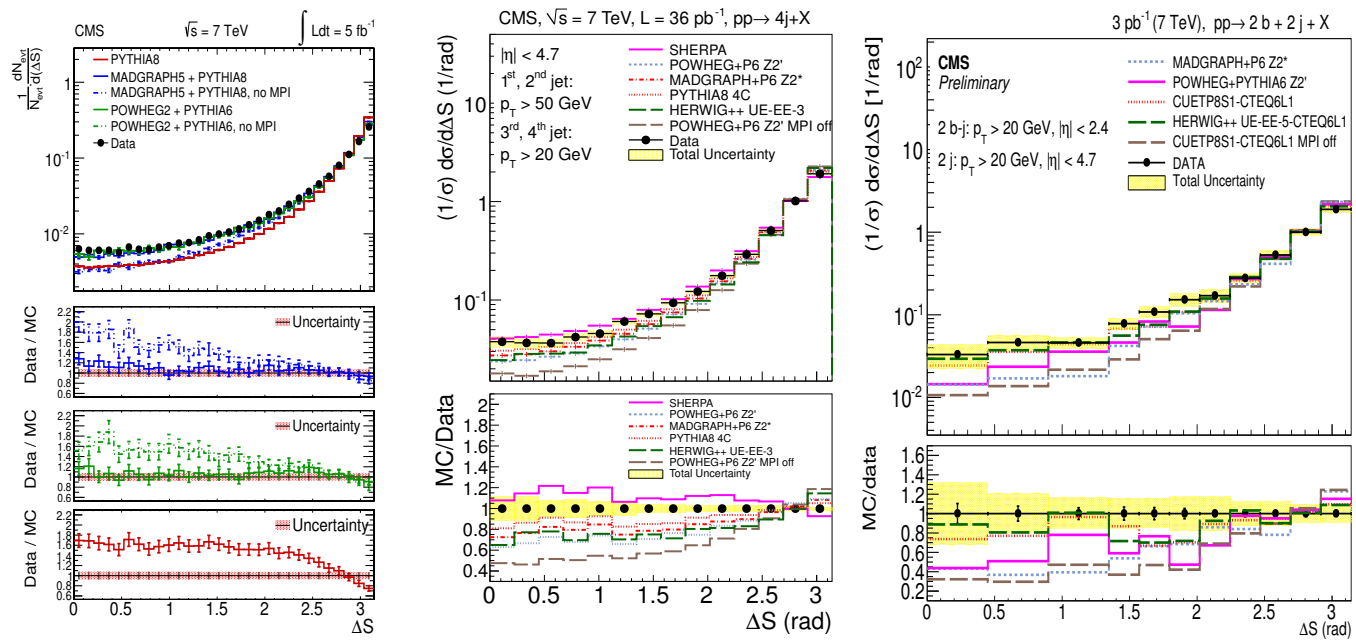

Figure 4. CMS data on the $\Delta \mathrm{S}$ observable for various measured final states: $\mathrm{W}+$ two-jet (left), four-jet (center), $\gamma+$ three-jet (right) channels.

In each of them, the measured cross sections have a similar shape with a falling distribution from correlated configurations at high values, down to uncorrelated jet topologies at low values. The measured cross sections have been also compared to predictions obtained with various MC event generators, implementing different matrix elements (ME) interfaced with parton shower (PS) and underlying event (UE) simulation. The PYTHIA 8 and HERWIG++ event generators simulate Leading Order (LO) $2 \rightarrow 2$ processes, SHERPA [26] and MADGRAPH [27] generate LO diagrams with higher number of partons in the final state, while POWHEG produces Next-to-Leading-Order (NLO) processes, with real and virtual corrections included in the ME. Predictions without the contribution of MPI are also considered in the comparison. All predictions include a simulation of parton-shower and underlying-event effects generated according to updated tunes.

In general, for all considered channels, predictions of multileg or NLO event generators are able to reproduce reasonably well the measured distributions. However, some room for improvement is available for the simulation of hard MPI contribution in the current models, in order to achieve a better description of the cross section at low values of $\Delta \mathrm{S}$. Predictions from event generators without the simulation of MPI do not reproduce the measured points, mainly in the region at low values of $\triangle \mathrm{S}$, where a DPS contribution is expected. This comparison clearly indicates the need for MPI in the current models to achieve a correct description of the DPS-sensitive observables. A quantitative extraction of the DPS contribution necessarily relies on the measurement of correlation observables and can be carried out through the so-called template [21] or the fitting [17] methods.

\section{Summary and conclusions}

The CMS experiment is very active in the research about many aspects concerning QCD and the understanding of the partonic behaviour in proton-proton collisions. A large set of jet measurements 
over different regions of phase space shows that current predictions based on the DGLAP evolution scheme are able to reproduce the data with a very good level of agreement. Investigations on observables sensitive to small- $x$ gluon dynamics did not give clear evidence of BFKL effects, yet. Various ideas and alternative approaches to tackle them in future measurements are under discussion.

The measurement of the integrated jet cross section performed with the CMS experiment as a function of the leading charged-particle and jet can be used as a probe of the transition from the perturbative to the non-perturbative region. The distributions, steeply falling at large transverse momenta and flattening out at $p_{T}$ values between 1 and $10 \mathrm{GeV}$, show effects of parton density saturation which are reproduced by models applying a cutoff of the partonic cross section at around 3-5 GeV. However, qualitative studies at generator level show that a better description of measurement at central and forward rapidities is achieved in case an increase at small- $x$ values is introduced for the gluon density.

Measurements sensitive to hard multiple interactions are available in many different physics channels. Observables investigating correlations in azimuthal angle between the objects selected in the considered final states can not be described by current models without implementing a simulation of multiple-parton interactions. These measurements can be used in the future to study the dependence of the double parton scattering contribution on the partonic initial state in terms of $x$ and flavour, and on the scale of the process.

\section{References}

[1] A. H. Mueller, Nucl. Phys. B 335 (1990) 115

[2] CMS Collaboration [CMS Collaboration], section at sqrt(s) = 8 TeV," CMS-PAS-SMP-14-001

[3] S. Chatrchyan et al. [CMS Collaboration], Phys. Rev. Lett. 107 (2011) 132001

[4] P. Kokkas [CMS Collaboration], PoS EPS -HEP2011 (2011) 277

[5] G. Altarelli and G. Parisi, Nucl. Phys. B 126 (1977) 298

[6] S. Chatrchyan et al. [CMS Collaboration], JHEP 1206 (2012) 036

[7] T. Sjostrand, S. Mrenna and P. Z. Skands, JHEP 0605 (2006) 026

[8] T. Sjöstrand, S. Mrenna and P. Z. Skands, Comput. Phys. Commun. 178 (2008) 852

[9] S. Gieseke, D. Grellscheid, K. Hamilton, A. Papaefstathiou, S. Platzer, P. Richardson, C. A. Rohr and P. Ruzicka et al., arXiv:1102.1672 [hep-ph]

[10] J. M. Campbell, R. K. Ellis, P. Nason and G. Zanderighi, JHEP 1308 (2013) 005

[11] H. Jung et al., Eur. Phys. J. C 70 (2010) 1237

[12] S. Chatrchyan et al. [CMS Collaboration], Eur. Phys. J. C 72 (2012) 2216

[13] J. R. Andersen and J. M. Smillie, JHEP 1106 (2011) 010

[14] C. Royon, AIP Conf. Proc. 1654 (2015) 070002

[15] V. Khachatryan et al. [CMS Collaboration], arXiv:1507.00233 [hep-ex]

[16] T. Pierog, I. Karpenko, J. M. Katzy, E. Yatsenko and K. Werner, Phys. Rev. C 92 (2015) 3, 034906

[17] CMS Collaboration [CMS Collaboration], CMS-PAS-GEN-14-001

[18] R. D. Ball et al. [NNPDF Collaboration], Nucl. Phys. B 877 (2013) 290

[19] P. Skands, S. Carrazza and J. Rojo, Eur. Phys. J. C 74 (2014) 8, 3024

[20] A. M. Cooper-Sarkar, PoS DIS 2014 (2014) 032

[21] S. Chatrchyan et al. [CMS Collaboration], JHEP 1403 (2014) 032

[22] S. Chatrchyan et al. [CMS Collaboration], Phys. Rev. D 89 (2014) 9, 092010

[23] CMS Collaboration [CMS Collaboration], CMS-PAS-FSQ-12-017

[24] CMS Collaboration [CMS Collaboration], CMS-PAS-FSQ-13-010 
[25] P. Gunnellini, DESY-THESIS-2015-010

[26] T. Gleisberg, S. Hoeche, F. Krauss, M. Schonherr, S. Schumann, F. Siegert and J. Winter, JHEP 0902 (2009) 007

[27] J. Alwall et al, JHEP 06 (2011) 128 